Research Paper

\title{
Outcome in Patients Resuscitated following Myocardial Infarction with Acute Kidney Injury
}

\author{
Vojko Kanic $^{\varpi}$, Robert Ekart and Zlatka Kanic \\ University Medical Center Maribor, Maribor, Slovenia. \\ $\triangle$ Corresponding author: Vojko Kanic, University Medical Center Maribor, Division of Internal Medicine, Department of Cardiology and Angiology, \\ Ljubljanska ulica 5, 2000 Maribor, Slovenia, Tel:+386 2321 2901, Fax:+386 2331 293, E-mail: vojko.kanic@guest.arnes.si. \\ (C) The author(s). This is an open access article distributed under the terms of the Creative Commons Attribution License (https://creativecommons.org/licenses/by/4.0/). \\ See http://ivyspring.com/terms for full terms and conditions.
}

Received: 2020.03.05; Accepted: 2020.05.19; Published: 2020.05.29

\begin{abstract}
Background: Data on acute kidney injury (AKI) in patients with myocardial infarction (MI) who underwent percutaneous coronary intervention (PCl) after cardiac arrest are scarce. The prevalence of $\mathrm{AKI}$, as classified by the Kidney Disease: Improving Global Outcomes (KDIGO) criteria; and its possible association with 30-day mortality were assessed.

Methods: Data on 6387 patients with MI, 342 (5.3\%) with out-of-hospital cardiac arrest or arrest immediately after admission before $\mathrm{PCl}$, were retrospectively analyzed. The $\mathrm{AKI}$ and no-AKI groups were compared. The 30-day mortality was determined.

Results: Ninety-three (27.2\%) patients suffered AKI. AKI KDIGO stages 1, 2 and 3 occurred in 45 (13.2\%), 8 (2.3\%) and 40 (11.7\%) patients, respectively. Higher mortality was found in AKI patients [56 $(60.2 \%)$ vs. no-AKI patients $32(12.9 \%)$; $<<0.0001]$. More patients died in the higher AKI KDIGO stages. In AKI KDIGO stages $1 / 2$ and stage 3, $20(37.7 \%)$ patients and $36(90.0 \%)$ patients died, respectively compared to 32 (12.9\%) no-AKI patients; $p<0.0001$.

AKI was the strongest predictor of 30-day mortality (adjusted OR $6.98 ; 95 \% \mathrm{Cl} 3.42$ to $14.23 ; \mathrm{p}<0.0001$ ). Other predictors were bleeding, cardiogenic shock, contrast volume-to-glomerular filtration rate ratio, and female sex. The adjusted OR for AKI KDIGO stages $1 / 2$ and stage 3 were $3.68 ; 95 \% \mathrm{Cl} 1.53$ to 8.32 ; $\mathrm{p}=0.002$ and $29.10 ; 95 \% \mathrm{Cl} 8.31$ to 101.88 ; $\mathrm{p}<0.0001$, respectively.

Conclusion: In patients resuscitated after $\mathrm{MI}$ undergoing $\mathrm{PCl}$, AKI had a deleterious impact on the prognosis. A graded increase in the severity of AKI according to the KDIGO definition was associated with a progressively increased risk of 30-day mortality.
\end{abstract}

Key words: acute kidney injury; cardiac arrest; resuscitation; myocardial infarction; outcome; percutaneous coronary intervention

\section{Introduction}

The incidence and survival of patients after out-of-hospital cardiac arrest (OHCA) vary 10-fold because of different definitions of OHCA and in-hospital cardiac arrest [1-7]. The different definitions might denote diverse patient populations (patients who died suddenly, all patients who died outside of hospital, those who were resuscitated, those who were attended by medical professionals, those with witnessed arrest, traumatic cardiac arrest, etc.) $[1,8]$, and, therefore, different survival rates would be expected. Survival rates following cardiac arrest vary from $0.6 \%$ to $25 \%$ [7]. Most cases of cardiac arrest $(55-78 \%)$ have a cardiac etiology. In "noncardiac" cardiac arrests, asphyxia and respiratory causes predominate $[7,9,10]$.

The complex combination of myocardial dysfunction and cerebral and whole-body ischemia in conjunction with the reperfusion response may lead to different combinations of multiorgan failure and significant morbidity and mortality [11]. Myocardial injury and circulatory failure account for most deaths in the first three days, but ischemic brain injury is the leading cause of in-hospital death [12]. The pathologic process that caused cardiac arrest in MI patients with 
OHCA may be resolved by coronary artery reperfusion and the survival rate in patients with percutaneous coronary intervention $(\mathrm{PCI})$ is, therefore, likely to be greater than in other OHCA patients $[11,13]$. Age, sex, time of cardiac arrest, and time to resuscitation are known predictors of survival in all OHCA patients [9]. In patients with cardiac arrest due to myocardial infarction (MI), age, female sex, increased serum glucose, ST-elevation MI, and initial admission to a hospital that was competent to perform primary PCI were associated with survival [14]. Data on additional risk factors and comorbidities associated with the outcome in MI patients with PCI are scarce [14].

Cardiopulmonary collapse after cardiac arrest and its aggressive treatment in MI patients are associated with increased risk of acute kidney injury (AKI) [11]. Myocardial infarction in combination with post-cardiac arrest myocardial dysfunction causes hypoperfusion of the kidneys [11,15]. PCI with possible atheroembolism, exposure to contrast media, bleeding, the need for cardiac support devices and drugs may further damage the kidney [15]. Additionally, the systemic ischemia/reperfusion response with inflammation and microcirculatory failure further aggravates AKI [11].

Different definitions of AKI in patients with OHCA of diverse etiology were used in previous studies. No data exist on the incidence of AKI according to the Kidney Disease Improving Global Outcomes (KDIGO) guidelines and its impact on outcomes in patients with cardiac arrest due to MI who underwent PCI [16]. We aimed to evaluate the prevalence of AKI, as classified by the KDIGO criteria, and to assess the impact of AKI on the outcome in patients with MI undergoing PCI who were resuscitated before hospitalization or immediately after admission but prior to PCI.

\section{Methods}

We performed a retrospective review of 6387 patients over 18 years of age with MI treated with PCI who were admitted to an academic tertiary care center between January 2007 and December 2016. We screened $423(6.6 \%)$ patients who were resuscitated before hospitalization or immediately after admission but prior to PCI. Patients on dialysis (9 patients) and patients who died before blood samples were taken or PCI was performed [53 (12.5\%) patients] were excluded. An additional 19 (4.2\%) patients who died within the first 24 hours were excluded, since it was assumed that there was no time for AKI to occur, leaving $342(81.0 \%)$ for further analysis (Figure 1).

Thrombolysis was not used. The group with AKI was compared with the group without AKI.

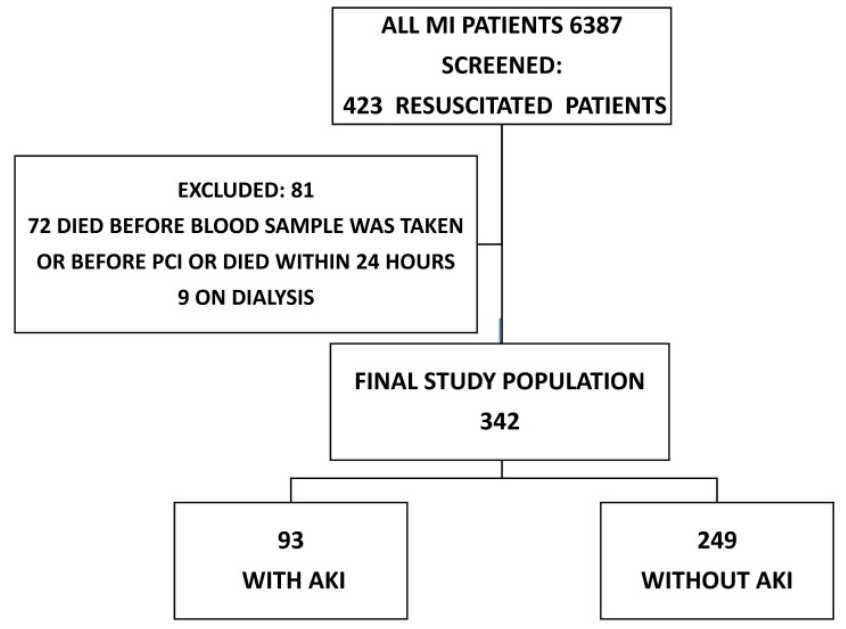

Figure 1. The diagram shows the number of MI patients screened, excluded and enrolled in the study. AKI: acute kidney injury; MI: myocardial infarction, $\mathrm{PCl}$ : percutaneous coronary intervention.

All-cause 30-day mortality and the incidence of AKI according to the KDIGO definition were assessed.

\section{Definitions}

Cardiac arrest was defined as cardiopulmonary collapse with associated loss of pulses requiring chest compressions or rescue shock by a health professional. We did not exclude patients on the basis of their initial cardiac rhythm or the duration of advanced cardiac life support.

All patients were Caucasians and were treated according to the guidelines for the management of MI $[17,18]$. The definition of ST-elevation MI was based on the ESC guidelines [17]. Therapeutic hypothermia with sedation/relaxation, cold fluid infusion and external cooling with ice packs was encouraged. The temperature was maintained between 32 and $34{ }^{\circ} \mathrm{C}$ for 24 hours.

Patient baseline creatinine concentrations were defined using one of three methods. The reference serum creatinine value was defined as a concentration obtained within 3-month period before admission. For patients without this reference value, we used the minimum of either serum creatinine at the time of admission, or we used a calculated serum creatinine concentration using the MDRD equation as recommended by the Acute Dialysis Quality Initiative (ADQI) in patients without chronic kidney disease $[2,19,20]$.

Renal dysfunction was defined as an estimated glomerular filtration rate (GFR) less than 60 $\mathrm{ml} / \mathrm{kg} / 1.73 \mathrm{~m}^{2}$. The Modification of Diet in Renal Disease Study formula was used for calculations of GFR [21]. Serum creatinine levels were determined on admission and after 24 and 48 hours. After the first 48 hours, serum creatinine levels were obtained at 
various time points, at the discretion of the treating physician.

AKI was defined as an increase in serum creatinine after PCI of $\geq 0.3 \mathrm{mg} / \mathrm{dl}(26.5 \mu \mathrm{mol} / \mathrm{L})$ in the first 48 hours [22]. Additionally, we determined AKI using the KDIGO criteria and staged for severity [16]. The Mehran score was calculated as proposed previously [23].

For calculations of AKI and mortality, we combined AKI KDIGO stages 1 and 2 (AKI KDIGO stage1/2) since there were only eight patients in AKI KDIGO stage 2 and there was no difference in 30-day mortality between AKI KDIGO stages 1 and 2 $(p=0.98)$.

The nonionic contrast agent iopamidol (concentration $370 \mathrm{mg} / \mathrm{ml}$ ) was used in our laboratory.

Renal protection with continuous infusion of saline $(1 \mathrm{ml} / \mathrm{kg} / \mathrm{h}$ during PCI and for the next 12 hours) was used. In patients with overt heart failure, the hydration rate was reduced at the discretion of the operator and/or attending physician.

Heart failure was defined according to the clinical criteria (bilateral pulmonary rales, $\mathrm{S}_{3}$ gallop, jugular venous distension) and/or ejection fraction $<30 \%$ and/or interstitial or alveolar edema requiring diuretic therapy on chest X-ray. The ventricular ejection fraction was assessed by bedside echocardiography in the first 48 hours after admission.

Cardiogenic shock on admission was defined according to clinical and hemodynamic criteria, including hypotension (systolic blood pressure $\leq 90$ $\mathrm{mm} \mathrm{Hg}$ for $\geq 30$ minutes or the need for supportive measures to maintain a systolic blood pressure of $>90$ $\mathrm{mm} \mathrm{Hg}$ ) and evidence of end-organ hypoperfusion.

The Bleeding Academic Research Consortium (BARC) bleeding criteria and BARC 3 a bleeding $(\mathrm{Hb}$ drop of 30-50 g/L or any transfusion) were used [24]. All medical records were obtained from the hospital information system to complete the data collection. The study was approved by the local medical ethics committee. Data on dates of death were provided by the Slovenian National Cause of Death Registry.

\section{Outcome}

The end point was 30-day all-cause mortality. The secondary end-point was the incidence of AKI.

\section{Statistical methods}

Distributions of continuous variables in the two groups were compared using either the 2-sample t-test or the Mann-Whitney test. Distributions of categorical variables were compared using the chi-square test. All p-values were two-sided; values less than 0.05 were considered statistically significant. We counted the end-point events that occurred during the follow-up period and compared their rates in the cohorts of patients with and without AKI. We used binary logistic regression to identify independent predictors of AKI and to calculate the adjusted odds of 30-day mortality. Binary logistic regression models were performed using the Enter mode. The models were adjusted for age, gender, diabetes, hypertension, bleeding, intra-aortic balloon pump, mechanical ventilation, cardiogenic shock, the contrast volume-to-estimated glomerular filtration rate ratio (contrast volume/GFR), renal dysfunction on admission, and AKI. Only mechanical ventilation and cardiogenic shock on admission were used as covariates. Data were analyzed with the SPSS 23.0 software for Windows (IBM Corp., Armonk, NY).

\section{Results}

\section{Descriptive patient data}

The clinical study encompassed 342 patients who were resuscitated out of hospital or immediately after admission before PCI. Information on the contrast volume used during PCI was available for $310(90.6 \%)$ patients. Other data were available for all patients.

Ninety-three $(27.2 \%)$ patients suffered AKI. AKI stage 1 occurred in $45(13.2 \%)$ patients, AKI stages 2 and 3 in eight $(2.3 \%)$ and $40(11.7 \%)$, respectively. Patients with AKI were older and were more likely to be mechanically ventilated and/or in cardiogenic shock at presentation. They more often suffered from diabetes and renal dysfunction but had less hyperlipidemia. They were more prone to bleeding, and a higher contrast volume/GFR ratio was observed. More intra-aortic balloon pumps were inserted. AKI patients were less liable to receive bivalirudin and double antiplatelet therapy. Higher peak troponin levels were usually seen together with lower ejection fraction. The Mehran score was more likely to be higher in the AKI group.

Therapeutic hypothermia was used in 141 $(41.2 \%)$. There was no difference in the rates of AKI between the temperature treatment groups $(52.7 \%$ in patients treated with therapeutic hypothermia compared to $38.6 \%$ in no-hypothermia patients; $\mathrm{p}=0.08$ ). Therapeutic hypothermia was not associated with AKI, (adjusted OR 0.63; 95\% CI 0.52 to 1.58). Additionally, therapeutic hypothermia did not affect the need for renal replacement therapy [7(3.5\% patients in the hypothermia group compared to $8(5.6 \%)$ in the no-therapeutic hypothermia group; $\mathrm{p}=0.48]$. 
Table 1. Basic patient characteristics

\begin{tabular}{|c|c|c|c|}
\hline & AKI; $N=93$ & No AKI; N = 249 & $\mathrm{p}$ \\
\hline Age, years $\infty$ & $66.0(12.5)$ & $61.8(11.8)$ & 0.029 \\
\hline Male gender, $\mathrm{N}(\%)^{*}$ & $67(72.0)$ & $177(71.1)$ & 0.89 \\
\hline ST-elevation MI, N (\%)* & $83(89.2)$ & $215(86.3)$ & 0.59 \\
\hline Diabetes, $\mathrm{N}(\%)^{*}$ & $25(26.9)$ & $35(14.1)$ & 0.01 \\
\hline Hypertension, $\mathrm{N}(\%)^{*}$ & $45(48.4)$ & $109(43.8)$ & 0.46 \\
\hline Hyperlipidemia, $\mathrm{N}(\%)^{*}$ & $18(19.4)$ & $86(34.5)$ & 0.008 \\
\hline Renal dysfunction, $\mathrm{N}(\%)^{*}$ & $46(49.5)$ & $53(25.3)$ & $<0.0001$ \\
\hline Cardiogenic shock, $\mathrm{N}(\%)^{*}$ & $37(39.8)$ & 48 (19.3) & $<0.0001$ \\
\hline Mechanical ventilation, $\mathrm{N}(\%)^{*}$ & $63(67.7)$ & $87(34.9)$ & $<0.0001$ \\
\hline Creatinine, $\mathrm{mgl} / \mathrm{dL}^{¥}$ & $1.20(0.94,1.65)$ & $0.94(0.79,1.20)$ & $<0.0001$ \\
\hline GFR, $\mathrm{ml} / \mathrm{kg} / 1.73 \mathrm{~m}^{2} ¥$ & $61.0(40.6,79.2)$ & $76.6(61.2,96.1)$ & $<0.0001$ \\
\hline Mehran score, $\mathrm{N} \infty$ & $12.9(5.4)$ & $8.66(5.2)$ & $<0.0001$ \\
\hline $\mathrm{BMI}, \mathrm{N} \infty$ & $27.8(4.5)$ & $27.4(4.5)$ & 0.49 \\
\hline
\end{tabular}

$\infty$ Mean (standard deviation); * Comparison made using the chi-square test; $¥$ Median (25th, 75th percentile).

BMI: body mass index; GFR: glomerular filtration rate; MI: myocardial infarction; $\mathrm{N}$ : number.

Table 2. Procedural characteristics of patients

\begin{tabular}{|c|c|c|c|}
\hline & AKI $(N=93)$ & No AKI $(\mathrm{N}=249)$ & $p$ \\
\hline Hypothermia, N (\%)* & $49(52.7)$ & $96(38.6)$ & 0.08 \\
\hline Contrast volume, $\mathrm{ml}^{¥}$ & $152.5(114.7,197.5)$ & $150.0(112.0,200.0)$ & 0.89 \\
\hline $\begin{array}{l}\text { Contrast volume-to-GFR } \\
\text { ratio }\end{array}$ & $2.66(1.67,4.19)$ & $1.90(1.34,2.90)$ & 0.001 \\
\hline Bivalirudin, $\mathrm{N}(\%)^{*}$ & $6(6.5)$ & $40(16.1)$ & 0.02 \\
\hline GPIIb/IIIa, N (\%)* & $58(52.4)$ & $133(53.4)$ & 0.14 \\
\hline $\begin{array}{l}\text { Dual antiplatelet therapy, } \mathrm{N} \\
(\%)^{*}\end{array}$ & $63(67.7)$ & $221(88.8)$ & $<0.0001$ \\
\hline PCI LMCA, N (\%) ${ }^{*}$ & $8(8.6)$ & $13(5.2)$ & 0.31 \\
\hline PCI LAD, N (\%)* & $45(46.4)$ & 115 (46.2) & 0.72 \\
\hline PCI LCX, N $(\%)^{*}$ & $26(28.0)$ & 57 (22.9) & 0.33 \\
\hline PCI RCA, N (\%)* & $21(22.6)$ & $72(28.9)$ & 0.28 \\
\hline Radial access, N (\%)* & $5(5.4)$ & $23(9.2)$ & 0.37 \\
\hline IABP, $\mathrm{N}(\%)^{*}$ & $11(11.8)$ & $13(5.2)$ & 0.054 \\
\hline $\begin{array}{l}\text { TIMI flow } 0 / 1 \text { after PCI, N } \\
(\%)^{*}\end{array}$ & $17(6.8)$ & $11(11.8)$ & 0.18 \\
\hline BARC 3a bleeding, $\mathrm{N}(\%)^{*}$ & $52(55.9)$ & $69(27.7)$ & $<0.0001$ \\
\hline Heart failure, $\mathrm{N}(\%)^{*}$ & $76(81.7)$ & $123(53.4)$ & $<0.0001$ \\
\hline Peak troponin, $(\mathrm{mol} / \mathrm{L})^{¥}$ & $40.92(8.3,84.3)$ & $24.26(6.7,65.6)$ & $<0.0001$ \\
\hline Ejection fraction, $(\%) \infty$ & $33.2(7.3)$ & $39.2(9.9)$ & $<0.0001$ \\
\hline Renal replacement therapy, & $14(15.1)$ & $0(0)$ & $<0.0001$ \\
\hline
\end{tabular}

$\mathrm{N}(\%)^{*}$

$\infty$ Mean (standard deviation);* Comparison made using the chi-square test; $¥$ Median (25th, 75th percentile).

AKI: acute kidney injury; BARC: Bleeding Academic Research Consortium; GFR: glomerular filtration rate; GPIIb/IIIa: GPIIb/IIIa receptor inhibitor; IABP. intra-aortic balloon pump; LAD: left anterior descending artery; LCX: circumflex artery; LMCA: left main coronary artery; N: number; PCI: percutaneous coronary intervention; RCA: right coronary artery; TIMI 0/1: TIMI grade flow after PCI: 0/1.

Renal replacement therapy (RRT) [14 (4.1\%) of all patients] was exclusively used in AKI patients. Continuous veno-venous hemofiltration was required by 10 patients, three patients were treated with a combination of veno-venous hemofiltration and temporary hemodialysis and one patient with temporary hemodialysis. Only one patient needed permanent RRT (hemodialysis). The risk of dying in 30 days was greater in patients who required RRT (71.4\% of patients with RRT died in 30 days compared to $20.6 \%$ of patients without RRT). However, in patients with AKI, RRT patients had similar rates of dying compared to no-RRT patients $(71.4 \%$ vs. $54.8 \%$; $\mathrm{p}=0.38$ ). The basic clinical and procedural characteristics of patients differed significantly between the groups, as shown in Tables 1 and 2.

\section{Mortality}

After 30 days, 88 (25.7\%) resuscitated patients had died. Patients who suffered AKI had a higher 30-day mortality [56 (60.2\%)) patients with AKI died compared to $32(12.9 \%)$ patients without AKI; $\mathrm{p}<0.0001]$. AKI independently predicted a worse 30-day mortality (adjusted OR 6.98; 95\% CI 3.42 to 14.23; $\mathrm{p}<0.0001$ ). Other predictors of mortality were bleeding, cardiogenic shock, contrast volume/GFR ratio and female sex (Table 3).

AKI KDIGO Stage $1 / 2$ patients had a higher unadjusted 30-day mortality [20 (37.7\%) patients with AKI 1/2 died compared to $32(12.9 \%)$ in the no-AKI group; $\mathrm{p}<0.0001)$ ].

The majority of AKI KDIGO stage 3 patients died within 30 days [36 $(90.0 \%)$ patients died compared to $32(12.9 \%)$ patients without AKI: $\mathrm{p}<0.0001)]$. The adjusted OR for AKI KDIGO stage $1 / 2$ and stage 3 were $3.68 ; 95 \%$ CI 1.53 to $8.32 ; \mathrm{p}=0.002$ and $29.10 ; 95 \%$ CI 8.31 to 101.88; $\mathrm{p}<0.0001$, respectively.

Table 3. Independent predictors of 30-day mortality

\begin{tabular}{llll}
\hline Variable & OR & $95 \%$ CI & $\mathrm{p}$ \\
\hline AKI & 6.98 & 3.42 to 14.23 & $<0.0001$ \\
Bleeding & 2.47 & 1.18 to 5.17 & $<0.0001$ \\
Cardiogenic shock & 2.82 & 1.38 to 5.75 & 0.004 \\
Female sex & 2.46 & 1.21 to 5.01 & 0.013 \\
Contrast volume-to-GFR ratio & 1.33 & 1.08 to 1.63 & 0.007 \\
\hline
\end{tabular}

AKI: acute kidney injury; GFR: glomerular filtration rate.

\section{Predictors of AKI}

Bleeding (OR 2.34; 95\% CI 1.30 to 4.19; $\mathrm{p}=0.004$ ), cardiogenic shock (OR 1.90; 95\% CI 1.01 to 3.58; $\mathrm{p}=0.046)$, mechanical ventilation (OR 2.91; 95\% CI 1.59 to $5.23 ; p=0.001$ ), and contrast volume/GFR ratio (OR 1.20; 95\% CI 1.01 to 1.41; $\mathrm{p}=0.033$ ) predicted AKI.

Interestingly, renal dysfunction did not independently predict AKI (adjusted OR 1.43; 95\% CI 0.63 to 2.49 ).

\section{Discussion}

Data on the association between AKI according to the KDIGO guidelines, and outcomes in patients with MI who underwent PCI after resuscitation are very limited [14]. In our study, almost seven percent of all MI patients undergoing PCI were resuscitated out of hospital or immediately after admission before PCI. We validated the prevalence of AKI based on the KDIGO criteria in unselected resuscitated patients with MI treated with PCI and their association with the outcome. The main findings of the present investigation are as follows: 
- Patients who suffered AKI after PCI had an almost seven times greater risk of dying in 30 days;

- AKI was the strongest independent predictor of mortality;

- A graded increase in the severity of AKI according to the KDIGO definition is associated with a progressively increased risk of 30-day mortality in these patients.

Our finding, that almost $30 \%$ of patients suffered AKI is consistent with a previous finding [2] but lower than the incidence of AKI in most previous observations of OHCA patients [12]. Our study group was specific because all patients suffered MI and all underwent PCI, which was not the case in previous reports, where different OHCA patients with or without PCI were included in the study [12]. The pathologic process that led to OHCA in our patients could have been resolved by PCI. Post-cardiac arrest myocardial dysfunction is both responsive to therapy and reversible, which could explain the better survival rate and the lower incidence of AKI [11].

We found AKI to be the strongest predictor of 30-day mortality in multivariate analysis, thus confirming the findings of a previous study in a similar population $[15,25]$. Yanta et al. found higher mortality in resuscitated patients with AKI, but AKI did not predict the outcome [2]. Unfortunately, any comparison is difficult due to the different study populations, different definitions of AKI and different treatment. Comparisons must be made in light of these differences. In our opinion, these differences could explain our result, which is in line with the previous finding [25].

Even patients with AKI KDIGO stage $1 / 2$ were almost four times more likely to die within 30-days compared to patients without AKI, while $90 \%$ of patients with AKI KDIGO stage 3 died within 30 days. A graded increase in the severity of AKI according to the KDIGO definition was associated with a progressively increased mortality risk. Our study confirmed the previous findings that bleeding, cardiogenic shock, and mechanical ventilation predict AKI $[15,26,27]$.

The contrast volume/GFR ratio was independently associated with AKI as found previously [28-30]. The contrast volume/GFR ratio probably better discriminates between the patients who are at higher risk of AKI than the contrast volume used or GFR alone [29,30]. The contrast volume used was similar in both groups and was not associated with AKI, while the contrast volume/GFR ratio differed significantly between the groups and predicted both AKI and 30-day mortality. Besides contrast volume and GFR, AKI in such patients after resuscitation is a consequence of the interplay of hemodynamic and nephrotoxic pathways, inflammation, and whole-body ischemia, in conjunction with the reperfusion response $[11,26]$.

Renal dysfunction on admission did not predict AKI. GFR on admission in the AKI group (median $61.0 \mathrm{ml} / \mathrm{min} / 1.73 \mathrm{~m} 2$ ) was above the defined threshold for renal dysfunction. This is consistent with the previous observation that renal dysfunction does not predict AKI in MI patients with cardiogenic shock and/or mechanical ventilation [15,31]. It is of note that $40 \%$ of patients with renal dysfunction in our analysis presented with cardiogenic shock, more than half of them were mechanically ventilated, and they were more liable to bleed after PCI (46\%), all of which predisposed to AKI [15,17,18,27,32,33].

There was a trend towards a higher rate of AKI in patients with therapeutic hypothermia $(p=0.08)$. It is noteworthy that patients treated with therapeutic hypothermia more often suffered STEMI $(91.8 \%$ vs. $83.1 \% ; \mathrm{p}=0.021)$, presented more often mechanically ventilated $(69.2 \%$ vs. $21.9 \%$; $<<0.0001)$ and in cardiogenic shock $(33.6 \%$ vs. $16.4 \%) ; \mathrm{p}<0.0001)$, and bled more (56.2\% vs. $20.2 \% ; \mathrm{p}<0.0001)$, which are all known risk factors for AKI $[30,34]$. Neither AKI nor RRT were associated with therapeutic hypothermia, as found previously $[35,36]$.

Female sex did not predict AKI but predicted a worse outcome, as seen previously [14].

Mechanical ventilation on admission predicted AKI but was not associated with mortality in our analysis. Patients with mechanical ventilation on admission presented more often in cardiogenic shock ( $32.7 \%$ vs. $18.8 \%$, p $=0.004)$, bled more $(52.0 \%$ vs. $22.4 \%$ $\mathrm{p}<0.0001)$, and had a greater contrast volume/GFR ratio ( 2.94 vs. $2.38 ; p=0.021$ ), which makes the higher mortality in these patients understandable. Although mechanical ventilation is a lifesaving procedure for critically ill patients, it is, at the same time, an indicator of a worse clinical outcome [37]. In resuscitated patients, mechanical ventilation is often only a temporary measure to overcome cardio-circulatory collapse and not a necessity because the patients are critically ill. These patients can often be weaned from mechanical ventilation shortly after the cause of cardiac arrest has been resolved. Only mechanical ventilation on admission was included in the multivariate analysis in the study. This difference may explain our finding.

Our findings have some potential clinical implications. We can easily identify factors that signify highly vulnerable patients on admission after OHCA. These are female sex, cardiogenic shock, and mechanical ventilation. Early commencement of 
preventive strategies like radial access to prevent bleeding, measures to ensure optimal hemodynamics, crystalloid infusions, and low contrast volume should be encouraged. Furthermore, adjustment of antithrombotic drugs and discontinuation of nephrotoxic drugs may ensure a better outcome. Additionally, consultation with a nephrologist early in the course of treatment might be beneficial $[15,16,26,28,30]$. In contrast to most studies, there was no difference in treatment between the groups with regard to reperfusion therapy. It is therefore questionable whether we can extrapolate our results to all OHCA patients.

In conclusion, we found that resuscitated patients with MI after PCI who suffered an AKI have a higher risk of dying. Preventive measures should be implemented early in the treatment.

\section{Limitations}

This study has several limitations. This was a relatively small, retrospective, single-center study. The data on the "time to resuscitation", "time to return of spontaneous circulation" and the "time between cardiac arrest and $\mathrm{PCI}^{\prime \prime}$ were not available for a sufficient number of patients to be considered in the evaluation, variables with known impact on AKI. We did not follow the data on possible normalization of serum creatinine. The exact hydration rate was not available in all patients because, in patients with overt heart failure, the hydration rate was reduced at the discretion of the operator and/or attending physician. Data on medications (ACE inhibitors, ARB, spironolactone), hyperuricemia and glucose were not collected. Moreover, data on a history of heart failure, coronary heart disease, and atrial fibrillation were not collected. The data on contrast volume were missing for some patients. However, patients without data on contrast volume were similarly distributed between the groups. Therefore, it is highly unlikely that this would have a significant impact on our result. We did not collect data for the Killip class or blood pressure, which are variables with a strong impact on AKI. Furthermore, we lack the data on urine output for 7 days. Calculations of eGFR were based on creatinine on presentation, which may not have been in a steady state, and thus may not be a true estimate of patients' baseline kidney function. For the calculations of eGFR, we used the MDRD equation, whose accuracy and reliability decrease with extreme eGFR values. Moreover, we were able to follow only all-cause mortality. Only the Caucasians with MI who underwent PCI, were included in the analysis. Therefore, the generalizability of our result is questionable.

\section{Conclusion}

AKI after PCI has a deleterious impact on the prognosis in patients resuscitated following MI. A graded increase in the severity of AKI according to the KDIGO definition is associated with a progressive increase in the risk of 30-day mortality.

\section{Abbreviations}

AKI: acute kidney injury; BARC: Bleeding Academic Research Consortium; BMI: body mass index; CI: confidence interval; ESC: European Society of Cardiology; GFR: glomerular filtration rate; GPIIb/IIIa: GPIIb/IIIa receptor inhibitor; IABP: intraaortic balloon pump; KDIGO: Kidney Disease Improving Global Outcomes; LAD: left anterior descending artery; LCX: circumflex artery; LMCA: left main coronary artery; MI: myocardial infarction; $\mathrm{N}$ : number; OHCA: out of hospital cardiac arrest; OR: odd ratio; PCI: percutaneous coronary intervention; RCA: right coronary artery; RRT: renal replacement therapy; TIMI: Thrombolysis in myocardial infarction.

\section{Acknowledgements}

\section{Ethics approval}

The study was approved by the Hospital Ethics Committee (UKC-MB-KME-24/19).

\section{Availability of data and materials' statement}

The datasets used and/or analyzed during the current study are available from the corresponding author on reasonable request.

\section{Funding}

This research did not receive any specific grant from funding agencies in the public, commercial, or not-for-profit sectors.

\section{Authors' contributions}

VK initiated the study, analyzed and interpreted the data, and wrote the initial manuscript. RE analyzed and interpreted the data and approved the final manuscript. ZK was involved in drafting or revising the manuscript, which was critically important for the intellectual content and approved the final manuscript. All the authors reviewed the manuscript.

\section{Competing Interests}

The authors have declared that no competing interest exists.

\section{References}

1. Berdowski J, Berg RA, Tijssen JG, et al. Global incidences of out-of-hospital cardiac arrest and survival rates: Systematic review of 67 prospective studies. Resuscitation 2010;11:1479-87. 
2. Yanta J, Guyette FX, Doshi AA, et al. Renal dysfunction is common following resuscitation from out-of-hospital cardiac arrest. Resuscitation. 2013;84:1371-4.

3. Bergman R, Hiemstra B, Nieuwland W, et al. Long-term outcome of patients after out-of-hospital cardiac arrest in relation to treatment: a single-centre study. Eur Heart J Acute Cardiovasc Care 2016;5:328-38.

4. Fordyce CB, Wang TY, Chen AY, et al. Long-Term Post-Discharge Risks in Older Survivors of Myocardial Infarction With and Without Out-of-Hospital Cardiac Arrest. J Am Coll Cardiol 2016;67:1981-90.

5. Wissenberg M, Folke F, Hansen CM, et al. Survival After Out-of-Hospital Cardiac Arrest in Relation to Age and Early Identification of Patients With Minimal Chance of Long-Term Survival. Circulation 2015;131:1536-45.

6. Forslund AS, Söderberg S, Jansson JH, et al. Trends in incidence and outcome of out-of-hospital cardiac arrest among people with validated myocardial infarction. Eur J Prev Cardiol 2013;20:260-7.

7. Hawkes C, Booth S, Ji C, et al. Epidemiology and outcomes from out-of-hospital cardiac arrests in England. Resuscitation 2017;110:133-40.

8. Barnard E, Yates D, Edwards A, et al. Epidemiology and aetiology of traumatic cardiac arrest in England and Wales - A retrospective database analysis. Resuscitation 2017;110:90-4.

9. Claesson A, Djarv T, Nordberg P, et al. Medical versus non-medical etiology in out-of-hospital cardiac arrest-Changes in outcome in relation to the revised Utstein template. Resuscitation 2017;110:48-55.

10. Noc M, Fajadet J, Lassen JF, et al. Invasive coronary treatment strategies for out-of-hospital cardiac arrest: a consensus statement from the European Association for percutaneous cardiovascular interventions (EAPCI)/stent for life (SFL) groups. EuroIntervention 2014;10:31-7.

11. Neumar RW, Nolan JP, Adrie C, Post-cardiac arrest syndrome: epidemiology, pathophysiology, treatment, and prognostication. A consensus statement from the International Liaison Committee on Resuscitation (American Heart Association, Australian and New Zealand Council on Resuscitation. Circulation 2008;118:2452-83.

12. Sandroni C, Dell'anna AM, Tujjar O, et al.Acute kidney injury after cardiac arrest: a systematic review and meta-analysis of clinical studies. Minerva Anestesiol 2016;82:989-99.

13. Callaway $\mathrm{CW}$, Schmicker RH, Brown SP, et al. Early coronary angiography and induced hypothermia are associated with survival and functional recovery after out-of-hospital cardiac arrest. Resuscitation 2014;85:657-63.

14. Couper K, Kimani PK, Gale CP, et al. Patient, health service factors and variation in mortality following resuscitated out-of-hospital cardiac arrest in acute coronary syndrome: Analysis of the Myocardial Ischaemia National Audit Project. Resuscitation 2018;124:49-57.

15. Marenzi G, Assanelli E, Campodonico J, et al. Acute kidney injury in ST-segment elevation acute myocardial infarction complicated by cardiogenic shock at admission. Crit Care Med 2010;38:438-44.

16. Kellum JA, Lamiere J, Aspelin P, et al. KDIGO clinical practice guideline for acute kidney injury. Kidney Int 2012;Suppl 2:1-138

17. Ibanez B, James S, Agewall S, et al. 2017 ESC Guidelines for the management of acute myocardial infarction in patients presenting with ST-segment elevation. European Heart Journal 2017;00:1-66.

18. Roffi M, Patrono C, Collet JP, et al. 2015 ESC guidelines for the management of acute coronary syndromes in patients presenting without persistent ST-segment elevation. European Heart Journal 2016:37:1257-315.

19. Hoste EA, Bagshaw SM, Bellomo R, et al. Epidemiology of acute kidney injury in critically ill patients: the multinational AKI-EPI study. Intensive Care Med 2015;41:1411-23.

20. Bellomo R, Ronco C, Kellum JA, et al. Acute renal failure - definition, outcome measures, animal models, fluid therapy and information technology needs: the Second International Consensus Conference of the Acute Dialysis Quality Initiative (ADOI) Group. Crit Care 2004;8:R204-12.

21. Levey AS, Coresh J, Greene T, et al. Expressing the Modification of Diet in Renal Disease Study Equation for Estimating Glomerular Filtration Rate with Standardized Serum Creatinine Values. Clinical Chemistry 2007:53:766 -72.

22. Mehta RL, Kellum JA, Shah SV, et al. Acute Kidney Injury Network: report of an initiative to improve outcomes in acute kidney injury. Crit Care 2007;11:R31.

23. Mehran R, Aymong ED, Nikolsky E, et al. A simple risk score for prediction of contrast-induced nephropathy after percutaneous coronary intervention: development and initial validation. J Am Coll Cardiol 2004;44:1393-9.

24. Mehran R, Rao SV, Bhatt DL, et al. Standardized bleeding definitions for cardiovascular clinical trials: a consensus report from the Bleeding Academic Research Consortium. Circulation 2011;23:2736-47.

25. Beitland S, Nakstad ER, Staer-Jensen $H$, et al. Impact of acute kidney injury on patient outcome in out-of-hospital cardiac arrest: a prospective observational study. Acta Anaesthesiol Scand 2016;60:1170-81.

26. Marenzi G, Cosentino N, Guastoni C. How to balance risks and benefits in the management of CKD patients with coronary artery disease. J Nephrol 2015;28:403-13.

27. Shacham Y, Steinvil A, Arbel Y. Acute kidney injury among ST elevation myocardial infarction patients treated by primary percutaneous coronary intervention: a multifactorial entity. J Nephrol 2016;29:169-74

28. Margolis G, Gal-Oz A, Letourneau-Shesaf S, et al. Acute kidney injury based on the KDIGO criteria among ST elevation myocardial infarction patients treated by primary percutaneous intervention. J Nephrol 2018;31:423-8.

29. Celik O, Ozturk D, Akin F, et al. Association Between Contrast Media Volume-Glomerular Filtration Rate Ratio and Contrast-Induced Acute Kidney
Injury After Primary Percutaneous Coronary Intervention. Angiology 2015;66:519-24

30. Kanic V, Kompara G, Suran D, et al. Impact of KDIGO-Defined Acute Kidney Injury on Mortality after Percutaneous Coronary Intervention for Acute Myocardial Infarction. Cardiorenal Med 2018;8:332-9.

31. van den Akker JPC, Bakker J, Groeneveld ABJ, et al. Risk indicators for acute kidney injury in cardiogenic shock. J Crit Care 2019;50:11-6.

32. Marenzi G, Cosentino N, Bartorelli AL. Acute kidney injury in patients with acute coronary syndromes. Heart 2015;101:1778-85.

33. Lazzeri C, Valente S, Chiostri M. Mechanical ventilation in the early phase of ST elevation myocardial infarction treated with mechanical revascularization. Cardiol J. 2013;20:612-7.

34. van den Akker JP, Egal M, Groeneveld AB. Invasive mechanical ventilation as a risk factor for acute kidney injury in the critically ill: a systematic review and meta-analysis. Crit Care. 2013;17(R):98.

35. Cornell TT, Selewski DT, Alten JA, et al. Acute kidney injury after out of hospital pediatric cardiac arrest. Resuscitation 2018;131:63-8.

36. Susantitaphong P, Alfayez M, Cohen-Bucay A, et al. Therapeutic hypothermia and prevention of acute kidney injury: a meta-analysis of randomized controlled trials. Resuscitation. 2012;83:159-67.

37. Kouraki K, Schneider S, Uebis R, et al. Characteristics and clinical outcome of 458 patients with acute myocardial infarction requiring mechanical ventilation. Results of the BEAT registry of the ALKK-study group. Clin Res Cardiol 2011;100:235-9. 